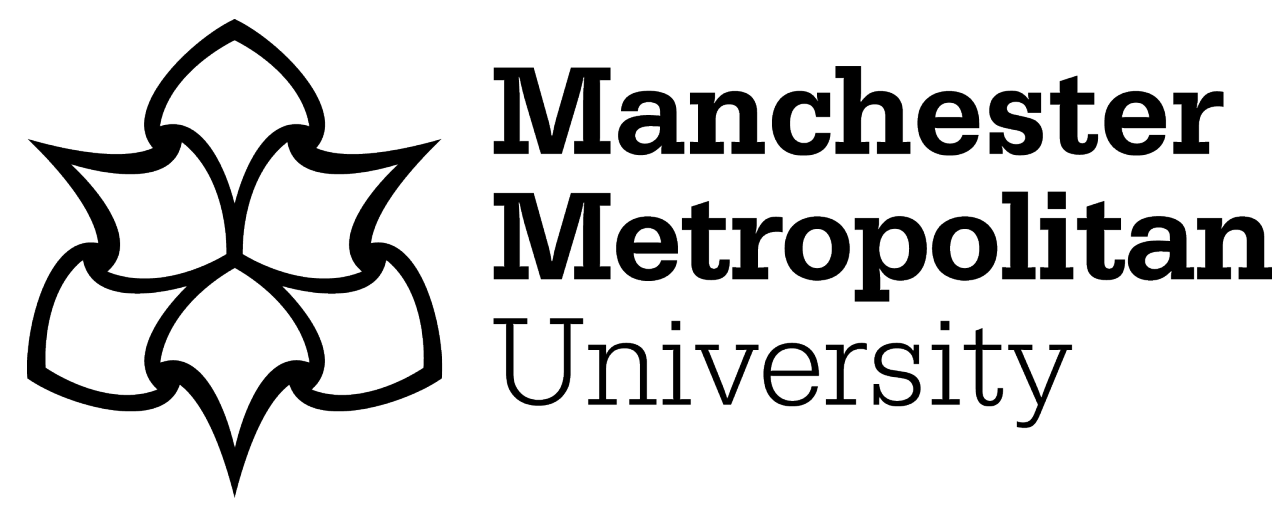

Gheth, W, Rabie, KM and Adebisi, B (2018) Impulsive Noise Modeling and Cancellation Strategies Over Power Line Channels. In: International Telecommunications Conference (ITelCon2017), 28 December 2017 - 29 December 2017, Istanbul, Turkey.

Downloaded from: https://e-space.mmu.ac.uk/621937/

Publisher: Springer

Please cite the published version 


\title{
Impulsive Noise Modeling and Cancellation Strategies over Power Line Channels
}

\author{
Waled Gheth, Khaled M. Rabie and Bamidele Adebisi \\ School of Engineering, Manchester Metropolitan University, UK, M1 5GD, \\ waled-ali.m.gheth@stu.mmu.ac.uk.
}

\begin{abstract}
Impulsive noise is one of the main impairments over power line channels. In this respect, this paper presents the available impulsive noise models and then reviews and compare several existing mitigation techniques proposed in the literature. These methods include multicarrier modulation, nonlinear preprocessors, multiple-input multiple-output, coding and iterative techniques. Interesting comparisons between these techniques and meaningful insights are presented and discussed.
\end{abstract}

Keywords: Coding, impulsive noise, multi-carrier modulation, power line communications.

\section{Introduction}

The principle of power line communication (PLC) is based on the use of the existing power lines in buildings and utility grid to carry data and electricity signals simultaneously. This technology has been around for some decades, but it has only been used for limited applications such as, home automation, public lighting and narrow band tele-remote relay applications. Recently, PLC technology has attracted much attention as almost every single building in the world can be reached by the existing electrical wiring. PLC also plays an important role in home networking applications due to the spreading of Internet and modern communication technologies.

On the other hand, there are several challenges when using this medium for high-data rate transmissions. Firstly, the frequency-selective nature of the channel introduces inter-symbol interference (ISI) which can significantly degrade bit error rate (BER) performance [1,2]. Secondly, there is the issue of electromagnetic compatibility (EMC) to other wireless systems in the surroundings. For this reason, there are regulatory limits for the maximum allowable power that a PLC modem can transmit [3] and this could result in very low signal-to-noise (SNR) values at the receiver. Thirdly, noise over power lines is one of the most destructive parameters which can disturb communication signals over PLC channels [4]. According to [4], the PLC noise is categorized into five types namely, colored background noise, narrow-band noise, periodic impulsive asynchronous to the mains frequency, periodic impulsive synchronous to the mains frequency and asynchronous impulsive noise. The first three types vary slowly over time 
and remain stationary over periods of up to minutes and sometimes for hours; therefore, they are summarized as background noise. However, the last two types are classified as impulsive noise which has short duration, random occurrence and high power spectral density (PSD). Communication signals over power lines can be severely degraded by impulsive noise. In this respect, this paper is dedicated to review and compare the existing techniques used to model and mitigate the impulsive noise.

The rest of the paper is organized as follows. Section 2 describes the noise modeling methods over power line channels. The impulsive noise mitigation techniques are discussed in Section 3 and section 4 concludes the paper.

\section{Impulsive Noise Modeling}

The statistical modeling of noise in power line channels has been an important interest to researchers recently. Plenty of research has been conducted to model impulsive noise $[5,6]$. Below, we discuss the most common impulsive noise models used in analyzing and evaluating the performance of PLC systems [7].

\subsection{Two-terms Gaussian Model}

For its simplicity, this model is widely used to characterize impulsive noise [7]; it is also known as two-component mixture-Gaussian model and is defined as

$$
\eta(t)=\varepsilon(t) n_{1}(t)+(1-\varepsilon(t)) n_{2}(t),
$$

where $\varepsilon(t)$ is a Bernoulli random process, $n_{1}(t)$ and $n_{2}(t)$ are independent Gaussian processes with zero mean and $\sigma_{b}^{2}$ and $K \sigma_{b}^{2}$ variances, respectively. The power of $n_{2}(t)$ is $K$ times greater than that of the background noise. The following formula describes the probability density function (PDF) of the total noise

$$
p_{\eta}(v)=(1-p) N\left(0, \sigma_{b}^{2}\right)+p N\left(0, K \sigma_{b}^{2}\right),
$$

where $N\left(0, \sigma_{b}^{2}\right)$ denotes the Gaussian distribution and $p$ is the probability occurrence of impulsive noise. The following formula is used to obtain the background noise variance

$$
\sigma_{b}^{2}=2 R_{I N} \int_{f_{1}}^{f_{2}} 10^{\left[R_{n b}(f)-30\right] / 10} \mathrm{~d} f\left[V^{2}\right],
$$

where $R_{I N}$ is the receiver input impedance and is usually equal to $50 \Omega, f_{1}=$ $1 \mathrm{MHz}$ and $f_{2}=100 \mathrm{MHz}$.

The following assumptions $\sigma_{b}^{2}=2.6456 \times 10^{-5}\left[V^{2}\right], K=100$ and $p=0.01$ are usually used to simulate the two Gaussian model, see e.g., $[8,9]$. 


\subsection{Middleton's Class-A Model}

Both the background and impulsive noise are incorporated in this model. It is worth mentioning that the communication channel that experiences class- $\mathrm{A}$ noise is also referred to as Additive White Class-A Noise channel (AWCN) [6]. The PDF of this noise model is defined as

$$
p_{\eta}(v)=\sum_{k=0}^{\infty} \frac{e^{-A} A^{k}}{k !} \frac{e^{v^{2} / 2 \sigma_{k}^{2}}}{\sqrt{2 \pi \sigma_{k}^{2}}}
$$

where

$$
\sigma_{k}^{2}=\left(1+\frac{1}{\Gamma}\right) \frac{k}{A}+\frac{\Gamma}{1+\Gamma} \sigma_{b}^{2},
$$

$\Gamma$ represents the background-to-impulsive noise ratio and $A$ is called the impulsive index.

A small number of terms in this series can be used to give acceptable approximations to this distribution. It was found that the cumulative sum can be cut off at the third term and the results obtained were very identical to the one found in the simple two-terms Gaussian model. Also, note that Middleton class-A noise becomes very close to Gaussian noise as the impulsive noise statistical characteristic becomes continuous when $A$ is large. On the contrary, highly structured impulsive noise is found when $A$ values are small. Similarly, same comments can be made when $\Gamma$ is changed while keeping $A$ constant.

\section{Impulsive Noise Cancellation Strategies}

In this sections, several impulsive noise mitigation techniques over PLC channels are presented and compared.

\subsection{Multi-carrier Modulation (MCM)}

A lot of work has been carried out trying to reduce the effect of impulsive noise. For example, the effect of impulsive noise on orthogonal frequency division multiplexing (OFDM) systems in radio communications was first analyzed in [10] where a closed-form expression for the probability of error was derived for two different scenarios, namely, single-carrier (SC) and MCM. It was found that MCM outperforms SC systems, more specifically when the noise power is moderate and the probability occurrence of noise is not too high. This was justified as follows: the effect of impulsive noise gets spread over several data symbols in the case of MCM systems whereas in SC systems, the impulsive noise will affect only one symbol. Furthermore, the author added that this advantage can turn into a disadvantage if the energy of impulsive noise exceeds a certain level. However, this study did not consider the effect of the channel. In [11], the BER performance of OFDM systems and the effect of multi-path over PLC channels were investigated by means of computer simulations. In [12], the BER performance of 
such systems under the effects of impulsive noise and multi-path was analyzed theoretically and closed-form formulas were obtained. It was concluded that the adverse effect of multi-path over PLC channels can be more serious than that of impulsive noise.

\subsection{Nonlinear Preprocessors}

Another mitigation technique is proposed in [13] where a nonlinear device is placed at the front end of the receiver. This technique is simple and easy to be implemented; due to these two reasons, this method is widely used in practice $[9,14,15]$. There are three different types of nonlinear preprocessors namely: blanking, clipping and combined blanking and clipping (Hybrid) whose basic principles are discussed below. These are also referred to as time domain (TD) mitigation techniques.

- Blanking non-linearity

$$
y_{k}=\left\{\begin{array}{ll}
r_{k}, & \left|r_{k}\right| \leq T_{b} \\
0, & \left|r_{k}\right|>T_{b}
\end{array} \quad k=0,1, \ldots, N-1\right.
$$

where $T_{b}$ is the blanking threshold, $y_{k}$ is the output of the nonlinear device and $r_{k}$ is its input.

- Clipping non-linearity [16]

$$
y_{k}=\left\{\begin{array}{ll}
r_{k}, & \left|r_{k}\right| \leq T_{c} \\
T_{c} e^{j \arg (r k)}, & \left|r_{k}\right|>T_{c}
\end{array} \quad k=0,1, \ldots, N-1\right.
$$

where $T_{c}$ is the clipping threshold.

- Hybrid (blanking/clipping) non-linearity [17]

$$
y_{k}= \begin{cases}r_{k}, & \left|r_{k}\right| \leq T_{c} \\ T_{c} e^{j \arg (r k)}, & T_{c}<\left|r_{k}\right| \leq T_{b} \quad k=0,1, \ldots, N-1 \\ 0, & \left|r_{k}\right|>T_{b}\end{cases}
$$

where $T_{b} \geq T_{c}$.

The assumption in this technique is that the received OFDM signal amplitude is conspicuously lower than the impulsive noise amplitudes. Therefore, any signals with amplitudes greater than a specified limit are assumed to be disturbed by impulsive noise [13]. However, using noise amplitude clipping or blanking techniques can enhance the communications performance. The received signal phase is not altered while the amplitude of the received signal is limited to a defined threshold by the nonlinear preprocessor [18]. Any OFDM sample that has amplitude lower than threshold passes without modifying its amplitudes as it is considered a clear OFDM signal. On the other hand, signal samples with amplitude above the threshold are clipped to $T_{c}$ in clipping technique or replaced with zero in blanking technique. Nevertheless, in the hybrid approach, signal 
samples that have amplitudes less than $T_{c}$ can pass without clipping or nulling. As $T_{c}$ is less than $T_{b}$, any sample with an amplitude above $T_{c}$ and less than $T_{b}$ is clipped to the clipping threshold while the OFDM sample whose amplitude is above both thresholds $T_{c}$ and $T_{b}$ is blanked. The concepts of the different TD techniques are illustrated in Fig. 1.

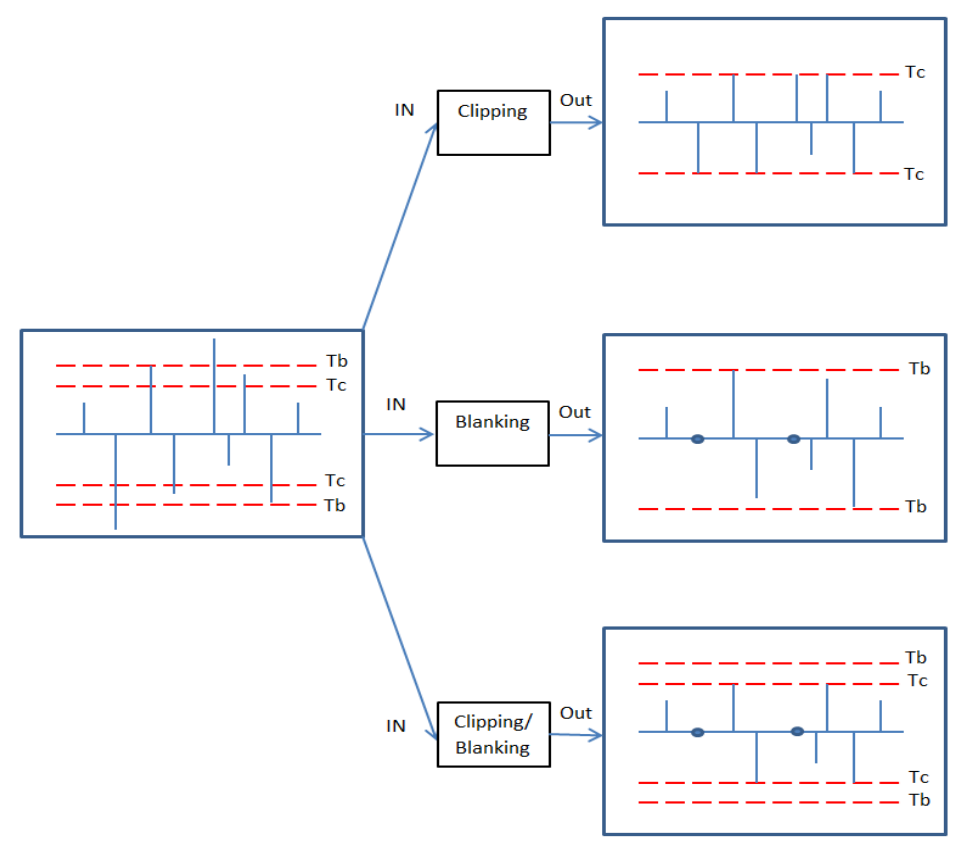

Fig. 1: TD impulsive noise mitigation techniques.

In [18], optimal $T_{c}$ and $T_{b}$ values were found and used to combat the impulsive noise over the narrow-band PLC systems. However, it was confirmed that the use of optimal threshold values for the hybrid scheme performs better than the use of either blanking or clipping separately. Specifically, a gain of $1.5 \mathrm{~dB}$ in the performance was achieved by combining clipping and blanking methods as was shown in [18]. Different impulsive noise scenarios were mitigated in [19], where the blanking and/or clipping techniques were used separately; improvement of $5 \mathrm{~dB}$ in the output SNR was achieved in the worst impulsive noise scenario when the hybrid method was used [19].

\subsection{Time and Frequency Domain (TFD) Techniques}

Unlike the aforementioned methods where the signal is processed before the OFDM demodulator, i.e., in the TD, the study in [20] proposed an algorithm that compensates for the impulsive noise and channel equalization after the 
OFDM demodulator, i.e., in the frequency domain (FD). This algorithm was investigated by means of simulation and a large performance improvement were achieved. Fig. 2 shows where the TD and the FD mitigation takes place.

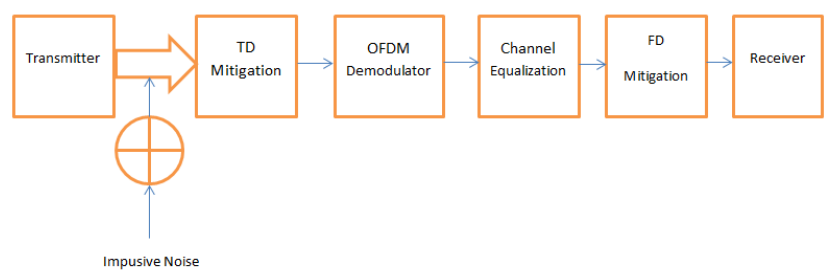

Fig. 2: TFD mitigation configuration.

A very small improvement in the SNR was achieved when a combination of amplitude clipping and channel equalization TFD technique was used for impulsive noise mitigation [21]. However, in this combined method, the amplitude clipping took place before the OFDM demodulator. The equalizer which was used as PLC channel compensator was located after the OFDM demodulator. The simulation results of this method showed that the system performance is enhanced by $0.7 \mathrm{~dB}$ as a maximum improvement in SNR [21]. Same impulsive noise mitigation technique was used in [19]. The OFDM signal is firstly passed through a clipping/blanking non-linearity TD processor then processed by the FD technique after it is passed through the demodulator and channel equalizer. A maximum of 5dB SNR improvement was reached with this method. This study considered different impulsive noise scenarios, namely, heavily, medium and weakly [21].

\subsection{Multiple Input Multiple Output (MIMO)}

MIMO in PLC refers to the concept of using multiple cables between the transmitter and the receiver and can be used to reduce the impact of several PLC channel impairments. MIMO techniques are mainly divided into:

Spatial Diversity Fading can be combated by the use of diversity. It is called transmit diversity if it is at the transmitter side and receive diversity if it is exploited at the receiver side.

Spatial Multiplexing This method is not supposed to strengthen the transmission; the data rate is increased when spatial multiplexing is used. The data is divided into separate streams and different cables are used to transmit these streams. Fig. 3 illustrates the main differences between MIMO in PLC and wireless channels. 


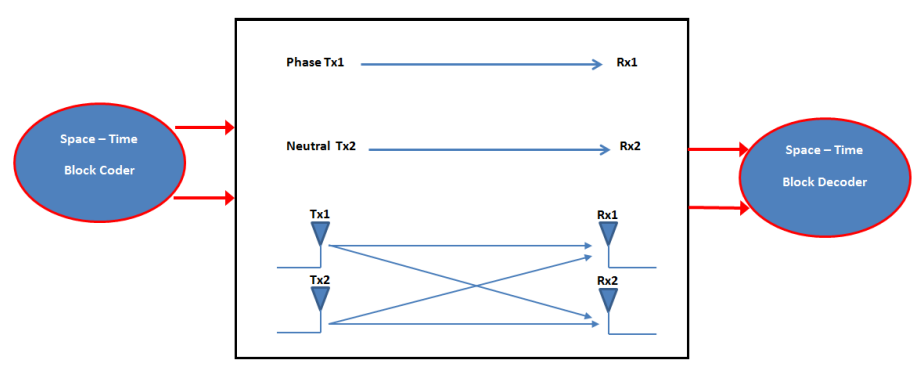

Figure 3: MIMO technology in PLC and wireless channels.

The performance of differential phase shift keying (DPSK), in the presence Middleton class-A noise, with two different diversity techniques, namely, equal gain combining (EGC) and selection combining (SC) in Ricean fading channels is investigated in [22]. It was found that when the proposed system is combined with linear block codes, the system performance can be further improved. Whereas in [23], closed-form expressions for BER performance are derived for two diversity schemes, namely, maximum ratio combining (MRC) and post detection combining (PDC) over Rayleigh fading channels using the two different impulsive noise models proposed in [24]. Unlike in Gaussian environments where MRC is the optimal, it was shown that PDC is more robust to impulsive noise than MRC scheme specially when the diversity order is high.

It is worth noting that there are some differences between the application of MIMO in power line channels and the wireless channel which can be seen in Fig. 3. Firstly, to obtain the input signal of every receiving antenna, the applications of MIMO in wireless communications combine the received signals from each transmitting antenna. On the other hand, as presented in [25], in order to simplify the transmission of the signals over the power lines, the wires ought to be completely isolated in PLC environments. Secondly, in PLC applications, the number of transmitting points must be equal to the number of receiving points [26]. In contrast, arbitrary number of antennas might be used in wireless applications. Thirdly, multiple transmitting and receiving antennas can be used in wireless communications whereas in PLC, a maximum number of three transmitting and receiving points are available. This means that for wireless communication with $N_{t}$ transmitting antennas and $N_{r}$ receiving antennas, a diversity order of $N_{t} N_{r}$ can be employed; while in PLC channels a maximum diversity order of three can be exploited.

Furthermore, many studies have investigated the use of space-time coding over PLC channels [27,28]. In [27], the author examined the performance of space-time block coding (STBC) with block interleaving in PLC channel contaminated with Middleton class-A impulsive noise and was compared to that in wireless channels; it was found that STBC is as promising as it is in wireless channels. In [26], the BER performance and channel capacity of STBC2x2 system are evaluated in a typical PLC environment, i.e., frequency selective channel 
and in the presence of AWCN. It was found that a gain of about $16 \mathrm{~dB}$ at BER of $10^{-5}$ can be achieved in comparison with the conventional system. As this study assumed complete isolation between the wires, it was presented that there is no capacity improvement in the proposed system.

All the studies above considered SC systems. As was presented in the previous section, MCM is more suitable for PLC channels. Space-frequency block coded (SFBC) [29] OFDM over a 3-phase power line network was studied in [28]. It was demonstrated that the proposed system provides a significant performance improvement, a gain of about $10 \mathrm{~dB}$ at symbol error rate of $10^{-3}$, compared to the conventional single-wire system.

STBC-OFDM over PLC channels was applied in [30] to improve the system reliability by applying symbol repetition technique. In [31], the performance of single-input single-output (SISO) and multiple-input single-output (MISO) techniques based on SC modulation was considered as well as the effect of feedback channels. It was demonstrated that MISO-SC systems with a feedback channel can outperform MISO-SC without a feedback channel and SISO-SC with and without feedback channels. In [32] MIMO-OFDM system was applied to PLC systems and the coupling effect between the conductors on the system capacity was evaluated experimentally.

\subsection{Coding}

Channel coding enables PLC nodes to detect and correct errors without the need for re-transmission, which may not be feasible in real-time based applications due to time delay restrictions. In general, convolutional and Reed-Solomon (RS) codes have been the main schemes adopted by narrow-band PLC standards. However, researchers have also evaluated the performance of other coding techniques such as the generalized array codes, Luby transform codes, bit-interleaved coded modulation with iterative decoding, row and column array codes. These codes have shown more robustness against power line channel impairments in comparison to the convolutional codes. Channel coding systems generally add redundancy to the original information to detect and correct errors. The performance of coded OFDM systems over power lines was first investigated in [33] by means of simulation. In this study, the authors examined two different channel coding methods namely, convolutional coding and RS coding. The author of [34] suggested adding bit-interleaving to the convolutionally coded OFDM and the BER performance was examined under three different impulsive noise scenarios based on the measurements of [4]. The simulation results showed that the combination of interleaving and convolutional coding can significantly minimize the effect of impulsive noise and a gain of up to $15 \mathrm{~dB}$ can be achieved at BER of $10^{-3}$

Turbo decoding for turbo codes in impulsive noise environments is discussed in [35] whereas the performance of double binary turbo coded OFDM system over PLC channels is analyzed in [36] where it was concluded that as the number of decoding process iterations increases, the BER performance is significantly improved. The study in [37] proposed using RS codes combined with M-ary 
modulation and showed that this can combat burst errors caused by impulsive noise even without applying interleaving. Quasicyclic low-density parity check (QC-LDPC) codes are implemented in PLC systems in the presence of Middleton class-A noise in [38].

It is true that the more robust the coding scheme employed is, the more link reliability is achieved; however, this will be attained at the expense of more computational complexity at the PLC modem resulting in more expensive chipset requirements.

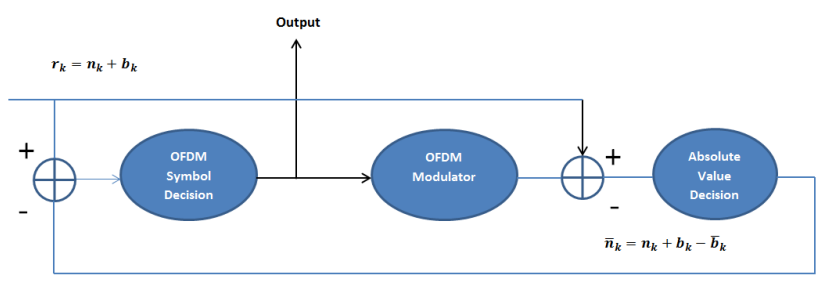

Figure 4: Iterative OFDM impulsive noise cancellation.

Iterative Impulsive Noise Reduction Several iterative algorithms have been proposed in the literature to mitigate impulsive noise [39]. For instance, Fig. 4 shows the iterative algorithm used in [39] which is based on the findings of [10] that MCM can combat the impulsive noise. In this algorithm, the output of the OFDM demodulator is re-modulated by the OFDM modulator and $\overline{b_{k}}$ is given. After that $\overline{b_{k}}$ is subtracted from the received signal $r_{k}$ and the result defines the estimated noise samples $\bar{n}_{k}$ which have large amplitudes. The estimated noise samples are subtracted from $r_{k}$. This process is repeated $n$ times depending on the number of iterations performed. Finally, after $n$ iterations, the resulting signal is passed through the OFDM demodulator. It was shown that when the number of iterations is 10 , this algorithm provides a gain of about $7 \mathrm{~dB}$ at BER of $10^{-3}$ compared to the conventional OFDM system, i.e., when the number of iteration $=0$.

Based on the above review of impulsive noise mitigation techniques, we can demonstrate a comparison between these techniques in terms of complexity, performance and data rate as in Table 1 .

\section{Conclusions}

In this paper, different impulsive noise modeling and mitigation techniques have been presented. The performance of the conventional communication schemes designed for Gaussian noise usually degrades drastically in non-Gaussian environments such as the PLC one. In general, MCM systems have better immunity 


\begin{tabular}{|l|c|c|c|c|}
\hline & Complexity & BER performance & Data rate & Transmission power \\
\hline \hline MCM & Medium & Good & Medium & Medium \\
\hline Nonlinear preprocessors & Low & Good & Medium & Medium \\
\hline MIMO & Medium & Good & Medium & Medium \\
\hline Coding & High & Very good & Low & Medium \\
\hline Iterative & High & Good & Medium & Medium \\
\hline
\end{tabular}

Table 1: Comparison between the various impulsive noise mitigation techniques.

to impulsive noise than SC systems. When the noise is very impulsive, nonlinear preprocessors become a very effective way of reducing the noise pulses. Furthermore, PLC systems can be made more resistant to such channel impairments by applying channel coding schemes. Increasing the transmit power to reduce the effect of such channels could solve the impulsive noise problem; however, this would create another problem, i.e. electromagnetic radiations. Therefore, there is a trade-off to which careful attention must be paid.

\section{Acknowledgment}

This research has been carried out within the "CityVerve: IoTs and Smart Cities Demonstrator" project funded by Innovate UK (102561).

\section{References}

1. D. .Anastasiadou and T. Antonakopoulos, "Multipath characterization of indoor power-line networks," IEEE Trans. Power Del., vol. 20, pp. 90-99, Jan. (2005). dio:10.1109/tpwrd.2004.832373.

2. M. Rozman, A. Ikpehai, B. Adebisi, and K. M. Rabie, "Channel characterisation of cooperative relaying power line communication systems," in Proc. Int. Symp. Commun. Systems, Networks and Digital Signal Process. (CSNDSP), pp. 1-5, Jul. (2016). dio:10.1109/csndsp.2016.7574000.

3. B. Adebisi and B. Honary, "Comparisons of indoor PLC emissions measurement results and regulation standards," Proc. Int. Symp. Power line Commun (ISPLC), pp. 319 - 324, Mar. (2006). dio:10.1109/isplc.2006.247482.

4. M. Zimmermann and K. Dostert, "Analysis and modeling of impulsive noise in broad-band powerline communications," IEEE Trans. Electromagn. Compat., vol. 44, pp. 249-258, Feb. (2002). dio:10.1109/15.990732.

5. D. Liu, E. Flint, B. Gaucher, and Y. Kwark, "Wide band AC power line characterization," IEEE Trans. Consum. Electron., vol. 45, pp. 1087-1097, Nov. (1999). dio: $10.1109 / 30.809186$.

6. D. Middleton, "Statistical-physical models of electromagnetic interference," IEEE Trans. Electromagn. Compat., vol. EMC-19, pp. 106 -127, Aug. (1977). dio:10.1109/temc.1977.303527.

7. S. Miyamoto, M. . Katayama, and N. Morinaga, "Performance analysis of qam systems under class-a impulsive noise environment," IEEE Trans. electromagn. Compat., vol. 37, pp. 260-267, May (1995). dio:10.1109/15.385891. 
8. K. M. Rabie and B. Adebisi, "Enhanced amplify-and-forward relaying in non-gaussian PLC networks," IEEE Access, vol. 5, pp. 4087-4094, (2017). dio:10.1109/ACCESS.2017.2680599.

9. B. Adebisi, K. M. Rabie, A. Ikpehai, C. Soltanpur, and A. Wells, "Vector OFDM transmission over non-gaussian power line communication channels," IEEE Systems Journal, vol. PP, no. 99, pp. 1-9, (2017). dio:10.1109/jsyst.2017.2669086.

10. M. Ghosh, "Analysis of the effect of impulse noise on multicarrier and single carrier QAM systems," IEEE Trans. Commun., vol. 44, pp. 145 -147, Feb. (1996). dio:10.1109/26.486604.

11. K. Kuri, Y. Hase, S. Ohmori, F. Takahashi, and R. Kohno, "Powerline channel coding and modulation considering frequency domain error characteristics," Proc. Int. Sympos. Power Line Commun. (ISPLC), pp. $221-225$, Oct. 2003.

12. Y. H. Ma, P. L. So, and E. Gunawan, "Performance analysis of OFDM systems for broadband power line communications under impulsive noise and multipath effects," IEEE Trans. Power Del., vol. 20, pp. 674-682, Apr. (2005). dio:10.1109/tpwrd.2005.844320.

13. S. Liu, F. Yang, W. Ding, J. Song, and Z. Han, "Impulsive noise cancellation for MIMO-OFDM PLC systems: A structured compressed sensing perspective," in IEEE Glob Commun. Conf. (GLOBECOM), pp. 1-6, Dec (2016). dio:10.1109/GLOCOM.2016.7841891.

14. K. Anoh, B. Adebisi, K. M. Rabie, M. Hammoudeh, and H. Gacanin, "On companding and optimization of OFDM signals for mitigating impulsive noise in power-line communication systems," IEEE Access, vol. PP, no. 99, pp. 1-1, (2017). dio:10.1109/access.2017.2747629.

15. A. Ikpehai, B. Adebisi, K. M. Rabie, M. Fernando, and A. Wells, "Energy-efficient vector OFDM PLC systems with dynamic peak-based threshold estimation," IEEE Access, vol. 5, pp. 10723-10733, (2017). dio:10.1109/access.2017.2709254.

16. H. A. Suraweera, C. Chai, J. Shentu, and J. Armstrong, "Analysis of impulse noise mitigation techniques for digital television systems," in Proc. 8th International OFDM Workshop, pp. 172 - 176, Sept. 2003.

17. S. V. Zhidkov, "Analysis and comparison of several simple impulsive noise mitigation schemes for OFDM receivers," IEEE Trans. Commun., vol. 56, pp. 5-9, Jan. (2008). dio:10.1109/tcomm.2008.050391.

18. M. Korki, N. Hosseinzadeh, H. L. Vu, T. Moazzeni, and C. H. Foh, "Impulsive noise reduction of a narrowband power line communication using optimal nonlinearity technique," in Australasian Telecommun. Networks and App. Conf. (ATNAC), pp. 1-4, Nov. 2011.

19. K. Al-Mawali, A. Z. Sadik, and Z. M. Hussain, "Joint time-domain/frequencydomain impulsive noise reduction in OFDM-based power line communications," in Australasian Telecommun. Networks App. Conf., pp. 138-142, Dec. 2008.

20. S. V. Zhidkov, "Impulsive noise suppression in ofdm-based communication systems," IEEE Trans. Consum. Electron., vol. 49, pp. 944 - 948, Nov. (2003). dio:10.1109/tce.2003.1261179.

21. Y.-H. Kim, K.-H. Kim, H.-M. Oh, K.-H. Kim, and S.-C. Kim, "Mitigation of effect of impulsive noise for OFDM systems over power line channels," in Proc. IEEE Int. Sympo. Power Line Commun. (ISPLC), pp. 386-390, Apr. 2008.

22. J. F. Weng and S. H. Leung, "On the performance of dpsk in rician fading channels with class a noise," IEEE Trans. Vehicular Technology, vol. 49, pp. $1934-1949$, Sep (2000). dio:10.1109/25.892596. 
23. C. Tepedelenlioglu and P. Gao, "Performance of diversity reception over fading channels with impulsive noise," IEEE International Conference on Acoustics, Speech, and Signal Processing, 2004. Proceedings. (ICASSP '04)., vol. 4, pp. 389392, May 2004.

24. P. A. Delaney, "Signal detection in multivariate class-a interference," IEEE Trans. Commun., vol. 43, pp. 365-373, Feb. 1995.

25. C. L. Giovaneli, J. Yazdani, P. Farrell, and B. Honary, "Application of space-time diversity/coding for power line channels," in Proc. of International Symposium on Power-Line Communications and Its Applications 2002 (ISPLC 2002), pp. 101105, Mar. 2002.

26. A. Papaioannou, G. Papadopoulos, and F. Pavlidou, "Performance of space-time block coding in powerline and satellite communications," IEEE JOURNAL OF COMMUNICATION AND INFORMATION SYSTEMS, vol. 20, no. 3, pp. 174181, (2005). dio:10.14209/jcis.2005.24.

27. A. P. G. D. Papadopoulos and F. N. Pavlidou, "Performance of space-time block coding over the power line channel in comparison with the wireless channel," in Proc. Int. Symp. Power Line Commun. (ISPLC), pp. 362-366, Apr. 2004.

28. C. L. Giovaneli, P. Farrell, and B. Honary, "Space-frequency coded ofdm system for multi-wire power communications," in Proc. Int. Sympos. Power Line Commun. (ISPLC), pp. 50-55, 2005.

29. K. Lee and D. Williams, "A space-frequency transmitter diversity technique for ofdm systems," IEEE Global Telecommunications Conference 2000, vol. 3, pp. 1473-1477, Nov. (2000). dio:10.1109/glocom.2000.891885.

30. Z. Quan and M. Ribeiro, "A low cost stbc-ofdm system with improved reliability for power line communications," in Proc. IEEE Int. Symp. Power Line Commun. (ISPLC), pp. 261 -266, Apr. (2011). dio:10.1109/isplc.2011.5764404.

31. F. de Campos, R. Machado, M. V. Ribeiro, and M. de Campos, "MISO singlecarrier system with feedback channel information for narrowband PLC applications," in Proc. IEEE Int. Symp. Power Line Commun. (ISPLC), pp. 301-306, Apr. 2009.

32. L. Hao and J. Guo, "A MIMO-OFDM scheme over coupled multi-conductor powerline communication channel," in Proc. IEEE Int. Symp. Power Line Commun. (ISPLC), pp. 198-203, Mar. 2007.

33. M. Babic, J. Bausch, T. Kistner, and K. Dostert, "Perfomance analysis of coded OFDM systems at statistically representative PLC channels," in Proc. IEEE Int. Symp. Power Line Commun. (ISPLC), pp. 104-109, (2006). dio:10.1109/isplc.2006.247445.

34. K. S. Al-Mawali and Z. M. Hussain, "Performance of bit-interleaved coded OFDM in power line communications with impulsive noise," pp. 49-53, Oct. 2009.

35. D. Umehara, H. Yamaguchi, and Y. Morihiro, "Turbo decoding in impulsive noise environment," vol. 1, pp. 194-198, Nov. (2004). dio:10.1109/glocom.2004.1377938.

36. E. C. Kim, S. S. Il, J. Heo, and J. Y. Kim, "Performance of double binary turbo coding for high speed PLC systems," IEEE Trans. Consumer Electronics, vol. 56, pp. 1211 -1217, Aug. (2010). dio:10.1109/tce.2010.5606248.

37. T. C. Chuah, "On reed solomon coding for data communications over powerline channels," IEEE Trans. Power Del., vol. 24, pp. 614 -620, Apr. (2009). dio:10.1109/tpwrd.2008.917667.

38. N. Andreadou, "Mitigation of impulsive noise effect on the PLC channel with QC-LDPC codes as the outer coding scheme," IEEE Trans. Power Del., vol. 25, pp. 1440-1449, Jul. 2010. 
39. H. Matsuo, D. Umehara, M. Kawai, and Y. Morihiro, "An iterative detection for OFDM over impulsive noise channel," Proc. Int. Symp. Power Line Commun. (ISPLC), Mar. 2002. 